\title{
Comment on "Efficacy and safety of percutaneous transforaminal endoscopic discectomy in the treatment of lumbar spinal stenosis combined with osteoporosis"
}

\author{
Jie Ding * ${ }^{1 *}$, Qiong-mei Han²
}

Dear Editor,

We read the article "Efficacy and safety of percutaneous transforaminal endoscopic discectomy in the treatment of lumbar spinal stenosis combined with osteoporosis" by Gu et al. ${ }^{1}$ with a great interest. The study found that percutaneous transforaminal endoscopic discectomy (PTED) is safe and effective in the treatment of lumbar spinal stenosis (LSS) combined with osteoporosis. However, some issues should be addressed from our point of view.

First, no published references were found for the evaluation of treatment efficacy. It is not a reasonable grouping for weekly exercise time. In the Results section, the study found that compared with the control group, the operation time, blood loss, and hospitalization duration in the PTED group were significantly decreased $(\mathrm{p}<0.05)$. The above sentence is a statistical misrepresentation. It should be stated that statistically significant difference was found for the operation time, blood loss, and hospitalization duration between two groups.

Second, another concern is the application of incorrect statistical methods in this study, because the treatment variable to assess the treatment effect is an ordered categorical data. The most appropriate statistical analysis methods for ordered categorical data should be nonparametric tests, for example, Mann-Whitney rank-sum test. However, the chi-square test was used to compare the treatment effects of two groups in this study.

\section{AUTHORS' CONTRIBUTIONS}

JD: Data curation, Formal analysis, Writing - original draft. QMH: Conceptualization, Writing - review and editing.

\section{REFERENCE}

1. Gu X, Zhu W, He H, Wang Z, Ding S, Guo G. Efficacy and safety of percutaneous transforaminal endoscopic discectomy in the treatment of lumbar spinal stenosis combined with osteoporosis. Rev Assoc Med Bras (1992). 2019;65(6):779-85. https://doi.org/10.1590/18069282.65 .6 .779

\footnotetext{
'Shanghai Jiaotong University, Changxing Branch of Xinhua Hospital Affiliated to Medical College, Department of Orthopaedics - Shanghai, China. ${ }^{2}$ Yankuang New Journey General Hospital, Department of Endocrinology - Jining, China.

*Corresponding author: jiangzuomeilang886@163.com

Conflicts of interest: the authors declare there are no conflicts of interest. Funding: none.

Received on August 11, 2021. Accepted on August 14, 2021.
} 